\title{
Breast Cancer-Associated Thrombotic Microangiopathy
}

\author{
Anne C. Regierer ${ }^{a} \quad$ Dagmar Kuehnhardt ${ }^{a}$ Carsten-Oliver Schulz ${ }^{a}$ Bernd Flath $^{b}$ \\ Christian F. Jehn ${ }^{a}$ Christian W. Scholz ${ }^{a} \quad$ Kurt Possinger $^{a} \quad$ Jan Eucker $^{a}$ \\ ${ }^{a}$ Department of Oncology and Hematology, Charité - Universitätsmedizin Berlin,

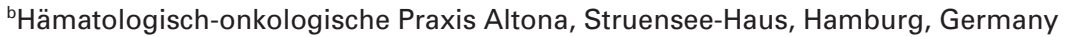

\section{Keywords}

ADAMTS13 - Bone marrow infiltration · Microangiopathic anemia $\cdot$ Thrombotic microangiopathy $\cdot$ Thrombotic thrombocytopenic purpura

\section{Summary}

Background: Thrombotic microangiopathy (TMA) is defined as thrombocytopenia and microangiopathic hemolytic anemia. Cancer-associated TMA, a rare but fatal condition, seems an entity distinct from classical thrombotic thrombocytopenic purpura (TTP)/hemolytic uremic syndrome (HUS). Patients and Methods: All patients with breast cancer-associated TMA treated at our institution between 2003 and 2008 were analyzed retrospectively. To elucidate pathophysiological mechanisms, we measured the serum activity of the metalloprotease ADAMTS13. Results: 8 patients were identified. All showed bone marrow infiltration of breast cancer as well as thrombocytopenia, schistocytes, and hemolytic anemia. ADAMTS13 activity was mildly decreased in 4/6 patients (20-108\%, normal range $30-120 \%$ ), but none showed severely low levels as is characteristic of classical TTP. 6 patients were treated with anthracycline-containing fractionated chemotherapy, 5/6 patients experienced partial response. Overall survival was 13 months. Fractionated chemotherapy was well tolerated. Conclusions: Cancer-associated TMA has an underlying mechanism different from classical TTP. While bone marrow infiltration might be of major relevance, ADAMTS13 deficiency seems to be an epiphenomenon. Fractionated chemotherapy resulted in higher remission rates and comparatively long survival.

\section{Introduction}

Thrombotic microangiopathy (TMA) is a rare but potentially devastating disease. It is defined as microangiopathic hemolytic anemia (MAHA) in combination with thrombocytopenia and signs of organ damage. These pathological features are included in different clinical syndromes, such as thrombotic

\section{KARGER}

Fax +497614520714

Information@Karger.de

www.karger.com
(C) 2011 S. Karger GmbH, Freiburg

$1661-3791 / 11 / 0066-0441 \$ 38.00 / 0$

Accessible online at:

www.karger.com/brc
Schlüsselwörter

ADAMTS13 · Knochenmarkinfiltration · Anämie, mikroangiopathische - Mikroangiopathie, thrombotische . Thrombotisch-thrombozytopenische Purpura

\section{Zusammenfassung}

Hintergrund: Thrombotische Mikroangiopathie (TMA) wird definiert als Thrombozytopenie mit mikroangiopathischer hämolytischer Anämie. Die zwar seltene, aber infauste Krebs-assoziierte TMA scheint eine andere Entität zu sein als die (das) verwandte klassische thrombotisch-thrombozytopenische Purpura (TTP)/hämolytisch-urämisches Syndrom (HUS). Patienten und Methoden: Alle an unserer Klinik zwischen 2003 und 2008 diagnostizierten Patienten mit Brustkrebs-assoziierter TMA wurden retrospektiv analysiert. Die Aktivität der Metalloprotease ADAMTS13 wurde ermittelt. Ergebnisse: 8 Patienten wurden identifiziert. Alle zeigten eine Knochenmarkinfiltration durch das Mammakarzinom sowie Thrombozytopenie, Fragmentozyten und hämolytische Anämie. Die ADAMTS13-Aktivität war bei $4 / 6$ Patienten leicht reduziert (20-108\%, Normalwert: 30-120\%), allerdings bei keinem so stark wie bei klassischer TTP. 6 Patienten wurden mit Anthrazyklin-haltiger fraktionierter Chemotherapie behandelt, $5 / 6$ zeigten eine partielle Response. Das Gesamtüberleben betrug 13 Monate. Die Chemotherapie wurde gut vertragen. Schlussfolgerungen: Der Krebs-assoziierten TMA liegt ein anderer Pathomechanismus zugrunde als der klassischen TTP. Während die leichte Reduktion der ADAMTS13-Aktivität nur ein Begleitphänomen zu sein scheint, spielt eine Knochenmarkinfiltration eine wichtige Rolle. Fraktionierte Chemotherapie zeigte eine hohe Wirksamkeit und resultierte in einer relativ langen Überlebenszeit.

thrombocytopenic purpura (TTP), hemolytic uremic syndrome (HUS), and also the HELLP syndrome in pregnancy. Recently, the pathophysiology of primary TTP has been widely elucidated. A severe deficiency of the metalloprotease ADAMTS13, either due to mutations or to autoantibodies, is presumed to be the main pathophysiological mechanism [1]. 
Secondary TMA can be triggered by many factors such as various drugs, bone marrow transplantation, infections, and malignant diseases. In cancer-associated TMA at least 2 different entities can be identified: chemotherapy-induced TMA and TMA induced directly by the cancer (non-chemotherapy-induced). Chemotherapy-induced TMA is described with a variety of known chemotherapeutic drugs such as mitomycin $\mathrm{C}$, bleomycin and cisplatin [2], but also with newer agents such as gemcitabine [3]. The incidence of chemotherapy-induced TMA varies between 0.1 and $10 \%$, depending on the drug. Only nonchemotherapy-induced TMA is the subject of this report.

Non-chemotherapy-induced TMA is mainly seen in latestage metastasized carcinomas. The underlying cancers are predominantly adenocarcinomas. There is increasing evidence that bone marrow infiltration, frequently seen in prostate, lung, breast, and ovarian cancer, is associated with TMA. However, many questions concerning pathophysiological aspects as well as the best therapeutic options are as yet unresolved.

Therefore, we have retrospectively analyzed all consecutively diagnosed patients with breast cancer-associated TMA. Pathophysiological aspects and therapeutic options are discussed. We present a promising therapeutic approach with fractionated chemotherapy resulting in longer survival compared to previously published reports.

\section{Methods and Patients}

We retrospectively analyzed all patients with breast cancer-associated TMA diagnosed at our institution between 2003 and 2008. Diagnosis was based on the presence of thrombocytopenia and Coombs-negative hemolytic anemia, as previously reported [4-6].

The patients were consecutively diagnosed and treated at our institution. Information recorded for each patient included age, tumor characteristics (size, regional lymph node status, histological characteristics, grading, hormone receptor status, and HER2/neu status), characteristics of metastases (localization and number of sites), therapy (palliative systemic treatment), and disease progression (time to progression and overall survival). The response evaluation criteria in solid tumors guidelines were used to assess the response to therapy. Laboratory findings, clinical signs and symptoms, treatment effects, and toxicity data were derived directly from the patient charts.

The following parameters of clinical routine were analyzed in the study: normal blood count, differential blood count, parameters of hemolysis (bilirubin, lactate dehydrogenase (LDH), haptoglobin), Coombs' test, renal and hepatic parameters, basic coagulation parameters (prothrombin time (PT), activated partial thromboplastin time (aPTT)).

In order to generate more insight into possible pathological mechanisms, we also measured the levels of von Willebrand factor (vWF) antigen and the serum activity of the vWF-cleaving protease, ADAMTS13. ADAMTS13 activity was measured by a modified method described by Furlan et al. [7].

\section{Results}

We identified 8 patients with breast cancer-associated TMA treated at our institution between 2003 and 2008, out of about 750 breast cancer patients. The baseline characteristics are

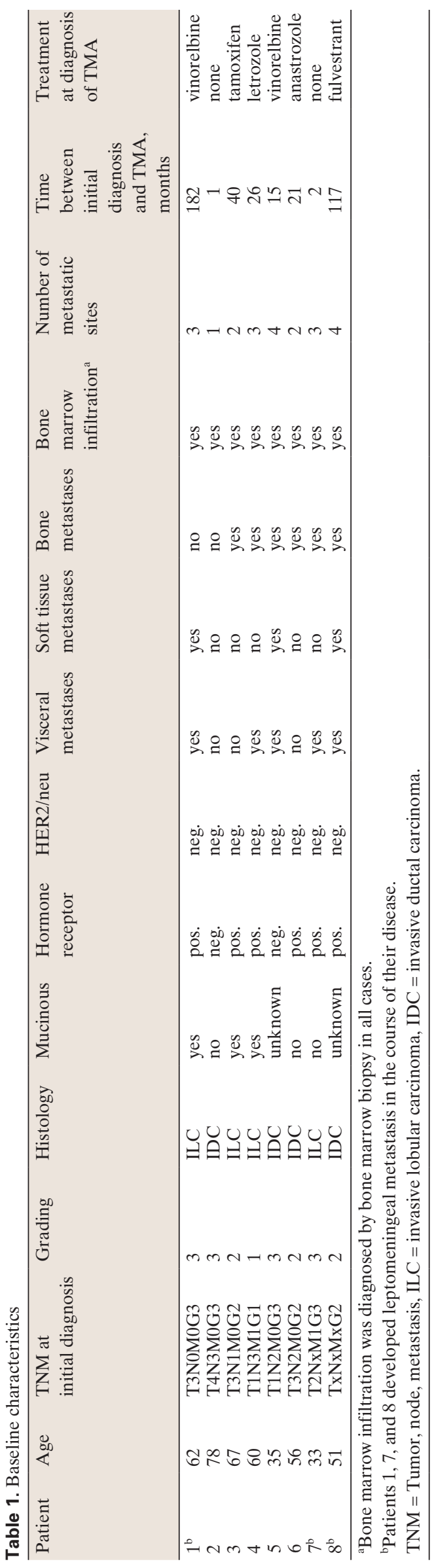

Regierer/Kuehnhardt/Schulz/Flath/Jehn/ Scholz/Possinger/Eucker 
Table 2. Signs and symptoms

\begin{tabular}{llllll}
\hline Patients & $\begin{array}{l}\text { Performance score } \\
\text { at diagnosis of TMA, } \%\end{array}$ & Cerebral symptoms & Renal signs & Signs of hemorrhage & Elevated temperature \\
\hline 1 & 50 & fatigue, confusion & none & petechiae & no \\
2 & 30 & confusion & none & none & no \\
3 & 70 & none & none & none & no \\
4 & 20 & confusion & none & none & no \\
5 & 60 & fatigue & none & none & no \\
6 & 70 & mild confusion & none & none & no \\
7 & 90 & none & none & mildly elevated creati- & hematoma \\
8 & 80 & headache & nine $(1.3 \mathrm{mg} / \mathrm{dl})$ & & \\
& & &
\end{tabular}

Table 3. Laboratory findings

\begin{tabular}{|c|c|c|c|c|c|c|c|}
\hline Patients & $\begin{array}{l}\text { Hemoglobin, } \\
\mathrm{mmol} / \mathrm{l}\end{array}$ & $\begin{array}{l}\text { Platelets, } \\
\times 10^{9} \mathrm{G} / 1\end{array}$ & $\begin{array}{l}\text { Leukocytes, } \\
\times 10^{9} \mathrm{G} / 1\end{array}$ & $\mathrm{LDH}, \mathrm{U} / \mathrm{l}$ & $\begin{array}{l}\text { Haptoglobin, } \\
\text { g/l }\end{array}$ & $\begin{array}{l}\text { vWF antigen }{ }^{\mathrm{a}} \text {, } \\
\%\end{array}$ & $\begin{array}{l}\text { ADAMTS13 } \\
\text { activity }^{\mathrm{b}}, \%\end{array}$ \\
\hline 1 & 6.09 & 88 & 16.50 & 4710 & $<0.04$ & $>420$ & not done \\
\hline 2 & 5.40 & 4 & 5.20 & 4068 & 0.08 & not done & not done \\
\hline 3 & 4.91 & 32 & 9.10 & 285 & 0.001 & 133 & 64 \\
\hline 4 & 2.98 & 32 & 17.50 & 1968 & $<0.04$ & 419 & 29 \\
\hline 5 & 5.53 & 15 & 6.30 & 4460 & 0.05 & $>420$ & 20 \\
\hline 6 & 6.21 & 95 & 10.10 & 2360 & $<0.04$ & 420 & 20 \\
\hline 7 & 4.97 & 11 & 19.23 & 780 & 0.07 & 401 & 28 \\
\hline 8 & 4.60 & 28 & 7.70 & 745 & $<0.04$ & 420 & 108 \\
\hline Median & 5.19 & 28 & 9.6 & 2164 & 0.06 & 419 & 28,5 \\
\hline
\end{tabular}

${ }^{a}$ Normal value: $50-150 \%$.

bNormal value: $30-120 \%$.

Table 4. Therapeutic and survival data

\begin{tabular}{|c|c|c|c|c|c|}
\hline Patient & Plasmapheresis & Chemotherapy & Best response & $\begin{array}{l}\text { Duration of response, } \\
\text { months }\end{array}$ & $\begin{array}{l}\text { Survival from onset } \\
\text { of TMA, months }\end{array}$ \\
\hline 1 & yes & A mono & not evaluable & not applicable & 1 (39 days) \\
\hline 2 & no & none & not applicable & not applicable & 0 (4 days) \\
\hline 3 & no & $\mathrm{AC}$ & $\mathrm{PR}$ & 13 & 29 \\
\hline 4 & no & FAC & PR & 8 & 12 \\
\hline 5 & no & FAC & PR & 7 & 13 \\
\hline 6 & no & $\mathrm{AC}$ & PR & 14 & 21 \\
\hline 7 & no & AC & PR & 9 & 15 \\
\hline 8 & no & $\mathrm{AC}$ & SD & 5 & 6 \\
\hline
\end{tabular}

outlined in table 1 . Interestingly, $50 \%$ of the patients were diagnosed with invasive lobular carcinoma, which is a higher proportion than in general breast cancer cohorts (10-20\%). We found evidence of mucine-producing carcinoma in 3 patients. All patients were widely metastasized, with visceral involvement in $5 / 8$ patients, and all showed bone marrow infiltration diagnosed by bone marrow biopsy. In 5 patients, the diagnosis of TMA was simultaneous to the diagnosis of distant metastases.

Clinical signs and symptoms at the onset of TMA are shown in table 2.3 patients presented in a poor performance status (PS $\leq 50)$, whereas 2 patients were only slightly impaired. In contrast to classical TTP/HUS, most of our patients showed only comparatively few signs of organ damage, although mild cerebral symptoms were found in the majority.

Table 3 outlines the laboratory findings. All patients had severe changes in their normal blood count. Normochrome anemia with a median hemoglobin level of $5.19 \mathrm{mmol} / \mathrm{l}$ was detected. Anemia was proven to be Coombs-negative hemolytic anemia with schistocytes in all cases. Thrombocyto- penia was also present in all cases, ranging from only a slight $\left(95 \times 10^{9} / \mathrm{l}\right)$ to a massive reduction $\left(4 \times 10^{9} / 1\right)$. The median platelet count at the time of diagnosis was $30 \times 10^{9} / 1$. Also, a severe elevation of the LDH level was found in $7 / 8$ patients. Surprisingly, creatinine was within normal limits in all but 1 patient.

The basic coagulation parameters were only slightly altered (median PT 68\% (normal value 70-120\%), median aPTT 34.05 (normal limits 26-40)) whereas the vWF antigen was severely elevated in 6/7 patients.

The activity of the ADAMTS13 protease was measured in 6 patients. A slight reduction was revealed in 4 cases but, interestingly, we did not find a severe reduction $(<5 \%)$ in any patient as would be expected in TTP.

The therapeutic strategy and the overall survival data are shown in table 4 . At the beginning of the study period (including patients 1 and 2), plasmapheresis was considered as standard therapy for suspected TTP in our institution, regardless of an underlying malignancy. The initiation of chemotherapy was considered secondary. In patient 1 , plasmapheresis was initiated immediately. Chemotherapy with adriamycin 
$20 \mathrm{mg} / \mathrm{m}^{2}$ weekly was started after 2 days. Although a short stabilization was achieved, the patient died 39 days after the diagnosis of TMA. Patient 2 presented in a very poor condition. After the diagnosis of TMA, plasmapheresis was considered. However, the patient deteriorated rapidly with an evolution towards multi-organ failure. Therefore, no specific therapy against TMA was initiated. The patient died 4 days after diagnosis.

Because of a growing body of evidence that patients with cancer-associated TMA benefit only slightly from plasmapheresis, we changed our therapeutic algorithm to initiate chemotherapy as early as possible and to withdraw plasmapheresis whenever possible (from patient 3 onwards).

In order to minimize the hematologic toxicity of standard chemotherapy, we used fractionated regimens according to the individual patient history. As anthracyclines are highly effective in breast cancer, we used adriamycin (A) either as monotherapy or in combination with cyclophosphamide (C) and 5-fluorouracil (F). The following dosages were used: A mono: $20 \mathrm{mg} / \mathrm{m}^{2} / \mathrm{d} 1$ weekly; AC: $20 / 200 \mathrm{mg} / \mathrm{m}^{2} / \mathrm{d} 1$ weekly; FAC: $200 / 20 / 200 \mathrm{mg} / \mathrm{m}^{2} / \mathrm{d} 1$ weekly.

The response rate with partial remission in 5/6 patients was surprisingly high. The responses included a clinically significant improvement of laboratory parameters (hemoglobin, thrombocytes, and LDH) in all and even normalization in 4 of the responding patients. The median duration of response was 8.5 months. The overall survival with a median of 13 months was higher than previously reported.

Inevitably, progression occurred in all patients. In 2 patients, the peripheral blood count deteriorated again, but the progression of the systemic disease, including mainly the progression in visceral metastatic sites, was the predominant aspect of the clinical deterioration. In 3 patients, a devastating leptomeningeal metastasis occurred at the time of progression, leading to death.

\section{Discussion}

Cancer-associated TMA is considered a rare but fatal condition in advanced cancer. The diagnosis of TMA is based on the findings of microangiopathic hemolytic anemia and thrombocytopenia. The combination of low erythrocyte and platelet counts is a common phenomenon in cancer patients, mostly due to the hematologic toxicity of chemotherapy. However, rare and serious conditions such as TMA should be taken into account, especially if the Coombs' test results are negative, and a thorough laboratory work-up should be undertaken to rule out the most obvious differential diagnoses. The most relevant sign of TMA in the differential blood count is the occurrence of schistocytes. However, these are not always present at the beginning of microangiopathy.

In contrast to primary TTP, in which a severe ADAMTS13 deficiency $(<5 \%)$ is the known pathophysiologic mechanism
$[1,7,8]$, the pathophysiology of cancer-associated TMA remains largely unknown. A variety of pathophysiologic considerations have been proposed, including tumor cell emboli, neoangiogenesis with fragile endothelium, mucin in mucinproducing tumors, and collision of disseminated carcinoma cells with erythrocytes. Also, injury to the endothelial cells of vessels in the bone marrow by direct tumor invasion has been discussed. We found very high vWF antigen levels in 6/7 patients, in accordance with Fontana et al. [9]. This can reflect endothelial damage, which might be of pathophysiologic significance.

The role of ADAMTS13 deficiency in cancer-associated TMA remains controversial. Although a severe deficiency has been described in some cases $[6,10]$, in most published cases a normal or only minimally reduced activity of the protease has been found $[5,6,9,11,12]$, which corresponds with our findings. Interestingly, there is evidence that cancer itself [13-15], as well as other severe medical conditions [16, 17], is associated with a moderate ADAMTS13 deficiency. However, in all of these cases the ADAMTS13 deficiency was mild, not reaching a rate as low as in TTP. These previous findings are in accordance with our data. It can be hypothesized that a mild ADAMTS13 deficiency is an epiphenomenon of the underlying cancer rather than a crucial pathophysiological mechanism of cancer-associated TMA. Another hint reinforcing this hypothesis is the low efficacy of plasma exchange therapy in cancer-associated TMA compared to TTP, in which the efficacy of this therapy depends on substituting the missing ADAMTS13 and/or on removing inhibitory antibodies against ADAMTS13.

It is worth noting that all our patients exhibited bone marrow infiltration, which is in concordance with the majority of published cases [4, 9-11, 18-26]. We therefore agree with the hypothesis of Chang and Naqvi [4] that bone marrow infiltration is highly associated with cancer-associated TMA. Whether bone marrow infiltration is a cause of TMA or an epiphenomenon needs further elucidation.

The optimal therapy for cancer-associated TMA is unknown. However, there is a growing body of evidence that immediate initiation of an effective antineoplastic treatment is of utmost importance. While plasma exchange therapy is the treatment of first choice for primary TTP, its benefit for cancer-associated TMA remains highly controversial [4-6, 11, $12,19,25-28]$. It is essential that plasma exchange therapy is further evaluated. However, there is increasing data that patients with cancer-associated TMA might not benefit from this invasive and expensive therapy.

The most important treatment option appears to be an efficient therapy of the underlying neoplastic disease. In line with this, several long-term survivors have been described, all of them with a complete or partial remission of the carcinoma [4, 6, 18-20, 22-25]. Due to this growing body of evidence, we have changed the treatment algorithm in our clinic. The initiation of chemotherapy became the primary goal, and omission 
of plasma exchange therapy was tolerated or even enforced. Our results with fractionated chemotherapy are surprisingly favorable and are in contrast to previous reports [4-6, 12]. The following reasons might explain these differences:

- In contrast to others, we describe only breast cancer patients, for whom various highly efficient chemotherapy options are known. Patients with metastatic breast cancer, although a palliative disease, have a longer overall survival than patients with many other metastatic malignancies that have been included in previous case series on cancer-associated TMA.

- In 5/8 patients the diagnosis of TMA was simultaneous to the diagnosis of distant metastases, and hence the fractionated chemotherapy induced for TMA was their first-line therapy. It is well known that efficacy is higher in the firstline setting than in the following lines.

- The fractionated chemotherapy regimens used in our patients were generally well tolerated. Therefore, dose reductions and delay of administration were uncommon. This might also explain the high effectiveness of chemotherapy in our patients.

Although the outcome of our patients was higher than previously reported, all patients inevitably experienced a progres- sion of breast cancer leading to death. Interestingly, while in 2 patients the peripheral blood count deteriorated again, in none of our patients was the recurrence of TMA the proposed cause of death. To the best of our knowledge, an association of TMA and leptomeningeal metastasis, as seen in 3/8 of our patients, has not been described before.

In conclusion, cancer-associated TMA is a devastating complication of malignant diseases. While a severe ADAMTS13 deficiency seems not to be the underlying pathophysiologic mechanism, bone marrow infiltration by carcinoma cells is strongly associated with TMA. The optimal management of the patients is not yet clear. We believe, however, that plasma exchange therapy has no or only minor therapeutic efficiency in cancer-associated TMA. In our opinion, it is crucial to induce effective antineoplastic therapy as soon as possible. Fractionated regimens seem a possible therapeutic way in order to minimize hematologic toxicity and to enable regular administration of chemotherapy.

\section{Disclosure Statement}

The authors declare no conflict of interest.

\section{References}

1 Brass L: vWF meets the ADAMTS family. Nat Med 2001;7:1177-1178.

2 Shahab N, Haider S, Doll DC: Vascular toxicity of antineoplastic agents. Semin Oncol 2006;33:121138

3 Humphreys BD, Sharman JP, Henderson JM, et al.: Gemcitabine-associated thrombotic microangiopathy. Cancer 2004;100:2664-2670.

4 Chang JC, Naqvi T: Thrombotic thrombocytopenic purpura associated with bone marrow metastasis and secondary myelofibrosis in cancer. Oncologist 2003;8:375-380

5 Francis KK, Kalyanam N, Terrell DR, et al.: Disseminated malignancy misdiagnosed as thrombotic thrombocytopenic purpura: A report of 10 patients and a systematic review of published cases. Oncologist 2007;12:11-19.

6 Oberic L, Buffet M, Schwarzinger M, et al.: Cancer awareness in atypical thrombotic microangiopathies. Oncologist 2009;14:769-779.

$\checkmark 7$ Furlan M, Robles R, Galbusera M, et al.: Von Willebrand factor-cleaving protease in thrombotic thrombocytopenic purpura and the hemolytic-uremic syndrome. N Engl J Med 1998;339:1578-1584.

$>8$ Tsai HM, Lian EC: Antibodies to von Willebrand factor-cleaving protease in acute thrombotic thrombocytopenic purpura. N Engl J Med 1998;339:1585-1594.

-9 Fontana S, Gerritsen HE, Kremer Hovinga J, et al.: Microangiopathic haemolytic anaemia in metastasizing malignant tumours is not associated with a severe deficiency of the von Willebrand factorcleaving protease. Br J Haematol 2001;113:100102.

10 Blot E, Decaudin D, Veyradier A, et al.: Cancerrelated thrombotic microangiopathy secondary to von Willebrand factor-cleaving protease deficiency. Thromb Res 2002;106:127-130.
11 Forman RB, Benkel SA, Novik Y, Tsai HM: Presence of ADAMTS13 activity in a patient with metastatic cancer and thrombotic microangiopathy. Acta Haematol 2003;109:150-152.

12 Elliott MA, Letendre L, Gastineau DA, et al.: Cancer-associated microangiopathic hemolytic anemia with thrombocytopenia: an important diagnostic consideration. Eur J Haematol 2010;85:4350 .

13 Böhm M, Gerlach R, Beecken WD, et al.: ADAMTS-13 activity in patients with brain and prostate tumors is mildly reduced, but not correlated to stage of malignancy and metastasis. Thromb Res 2003;111:33-37.

14 Mannucci PM, Karimi M, Mosalaei A, et al.: Patients with localized and disseminated tumors have reduced but measurable levels of ADAMTS-13 (von Willebrand factor cleaving protease). Haematologica 2003;88:454-458.

15 Koo BH, Oh D, Chung SY, et al.: Deficiency of von Willebrand factor-cleaving protease activity in the plasma of malignant patients. Thromb Res 2002;105:471-476.

16 Kremer Hovinga JA, Zeerleder S, Kessler P, et al.: ADAMTS-13, von Willebrand factor and related parameters in severe sepsis and septic shock. J Thromb Haemost 2007;5:2284-2290.

17 Mannucci PM, Vanoli M, Forza I, et al.: Von Willebrand factor cleaving protease (ADAMTS-13) in 123 patients with connective tissue diseases (systemic lupus erythematosus and systemic sclerosis). Haematologica 2003;88:914-918.

18 Lin YC, Chang HK, Sun CF, Shih LY: Microangiopathic hemolytic anemia as an initial presentation of metastatic cancer of unknown primary origin. South Med J 1995;88:683-687.
19 Abdel Samie A, Sandritter B, Theilmann L: [Severe microangiopathic hemolytic anemia as first manifestation of a CUP syndrome. Rapid hematologic remission under polychemotherapy]. Med Klin 2004;99:148-153.

20 von Bubnoff N, Sandherr M, Schneller F, Peschel C: Thrombotic thrombocytopenic purpura in metastatic carcinoma of the breast. Am J Clin Oncol 2000;23:74-77.

21 Ataga KI, Graham ML: Microangiopathic hemolytic anemia associated with metastatic breast carcinoma. Am J Hematol 1999;61:254-255.

22 Narita M, Nakao K, Ogino N, et al.: A case of microangiopathic hemolytic anemia associated with breast cancer: improvement with chemoendocrine therapy. Breast Cancer 1997;4:39-42.

23 Nordstrom B, Strang P: Microangiopathic hemolytic anemias (MAHA) in cancer. A case report and review. Anticancer Res 1993;13:1845-1849.

24 Antman KH, Skarin AT, Mayer RJ, et al.: Microangiopathic hemolytic anemia and cancer: a review. Medicine 1979;58:377-384.

25 Lee JL, Lee JH, Kim MK, et al.: A case of bone marrow necrosis with thrombotic thrombocytopenic purpura as a manifestation of occult colon cancer. Jpn J Clin Oncol 2004;34:476-480.

26 Spoormans I, Altintas S, Van den Brande J, et al.: Purpura in a patient with disseminated breast cancer: a rapidly progressive cancer-related thrombotic thrombocytopenic purpura. Ann Oncol 2008;19:1204-1207.

27 Kwaan HC, Gordon LI: Thrombotic microangiopathy in the cancer patient. Acta Haematol 2001;106:52-56.

28 Werner TL, Agarwal N, Carney HM, Rodgers GM: Management of cancer-associated thrombotic microangiopathy: what is the right approach? Am J Hematol 2007;82:295-298. 